\title{
A Bi-level IHS Transform for Fusing Panchromatic and Multispectral Images
}

\author{
Navaneeth K. Ramakrishnan and Philomina Simon \\ Department of Computer Science, University of Kerala, Thiruvananthapuram \\ \{navaneeth.kr,philomina.simon\}@gmail.com
}

\begin{abstract}
In this paper, a new method for Panchromatic and Multispectral satellite image fusion is proposed. The major challenge of a fusion algorithm is to improve the spatial and spectral qualities of the fused image. But the spatial and spectral qualities are inversely proportional; we cannot improve either quality above particular range without losing visual quality, and most of the current methods do not take into consideration about visual quality. The proposed method tries to improve the spatial and visual quality with reduced spectral distortion using a Bi-Level Intensity Hue Saturation transform. Proposed method is rigorously tested over QuickBird and IKONOS satellite images and the experimental results shows that our method produces high visual quality fused images with a good spatial and spectral quality levels compared with existing methods.
\end{abstract}

Keywords: Bi-Level IHS, Image Fusion, QuickBird, IKONOS, Visual Quality, Remote Sensing.

\section{Introduction}

Satellite images are widely used in remote sensing applications. Earth observing satellites like QuickBird and IKONOS provide two types of images; Multispectral (MS) and Panchromatic (PAN) images. MS images have high spectral resolution and PAN images have very good spatial resolution. It is desirable to have the best possible spatial resolution in order to find fine features on the Earth's surface for most remote sensing applications. Moreover, a high spectral resolution is also required to discriminate among different ground covers. Most of these remote sensing applications need human intervention for feature detection and classification of land area, so that visual quality of the fused image is also an important factor. However, for certain applications, there is a need to improve the spatial resolution of the MS images. Improving the spatial quality of the MS image is usually called pansharpening. The main issue in the pansharpening is the inverse proportionality between spectral and spatial quality. When we try to improve one quality the other quality become reduced. A good fusion algorithm must attain a good spectral and spatial quality level, that is, good spatial quality with less spectral distortion. Mapping and surveillance application demand not only high spatial and spectral quality images but also the good visual quality for images. Most of the methods are not concerned about the visual quality of the fused image. 
Within the well known spatial domain fusion methods IHS[1], Brovey[2] and PCA[2] methods achieve fine spatial quality but generate spectral distortions. Another family of methods using wavelets produces much less spatial quality images than those fused by the Brovey, IHS, and PCA methods. Some of the new transformations such as courvelet, contourlet[3][4] and NSCT[5][6] are proposed to overcome this weakness. The contourlet has many advantages over curvelet. A. G. Mahyari et al[5]., proposed a new method for identifying a best threshold for fusing MS and PAN images. But algorithm failed to provide good spatial quality. Then researchers try to combine spatial and transform domain fusion methods. Hybrid methods like NSCT and IHS[6], Bilateral filter and IHS[7] are try to combine good qualities of both techniques, and able to achieve good spectral quality but the sharpness of the fused image is lost, also the time complexity is also very high.

Many new less complex methods are introduced to reduce the spectral distortion happened during the image fusion using IHS transform. Within that Adaptive-IHS[8] and Improved-IHS[9] provide good spectral quality but the image is visually distorted. The new IHS fusion by Choi[10] able to preserve the spectral and spatial qualities up to a good level, but lose of sharpness reduces the visual quality. In this paper out objective is to achieve a good visual quality by fusing PAN spatial details along with color details of MS.

\section{Bi-level IHS Fusion Algorithm}

A good fusion algorithm should improve two inversely proportional qualities, ie., spatial and spectral, which is not possible simultaneously. So we are trying to achieve a good spatial and spectral quality levels along with good visual quality using a BiLevel IHS algorithm. The Bi-Level IHS method has two levels of IHS based fusion algorithms, within that the first level algorithm brings spatial information to the fused image using fast IHS and new adaptive averaging; and the second level algorithm fuse spectral details to the MS image.

The first level of proposed fusion method uses a histogram equalized PAN image for the fast IHS transform, and that can be implemented as follows,

$$
\mathrm{PAN}_{\mathrm{eq}}=\mathrm{PAN} * \frac{\sigma_{\mathrm{I}}}{\sigma_{\mathrm{PAN}}}
$$

where, $\sigma_{\text {I }}$ standard deviation of intensity component and $\sigma_{\text {PAN }}$ is the standard deviation of PAN image.

$$
\begin{aligned}
& {\left[\begin{array}{l}
R_{I H S}^{\prime} \\
G_{I H S}^{\prime} \\
B_{I H S}^{\prime}
\end{array}\right]=\left[\begin{array}{l}
R+\left(P^{2} N_{e q}-I\right) \\
G+\left(P_{\text {AN }}-I\right) \\
B+\left(P_{\text {AN }}-I\right)
\end{array}\right]} \\
& I=(R+G+B+N I R) / 4
\end{aligned}
$$

The next step in first level operation is to apply the adaptive averaging function. The adaptively added MS components and PAN details helps to improve the spatial quality and reduces the spectral distortion. The averaging function is: 


$$
\left[\begin{array}{c}
\mathrm{R}_{\mathrm{IHS}}^{\prime \prime} \\
\mathrm{G}_{\mathrm{IHS}}^{\prime \prime} \\
\mathrm{B}_{\mathrm{IHS}}^{\prime \prime}
\end{array}\right]=\left[\begin{array}{c}
\left(\mathrm{R}_{\mathrm{IHS}}^{\prime}+\mathrm{PAN}_{\mathrm{eq}}+\mathrm{R} * \alpha_{1}\right) /\left(\alpha_{1}+2\right) \\
\left(\mathrm{G}_{\mathrm{IHS}}^{\prime}+\mathrm{PAN}_{\mathrm{eq}}+\mathrm{G} * \alpha_{2}\right) /\left(\alpha_{2}+2\right) \\
\left(\mathrm{B}_{\mathrm{IHS}}^{\prime}+\mathrm{PAN}_{\mathrm{eq}}+\mathrm{B} * \alpha_{3}\right) /\left(\alpha_{3}+2\right)
\end{array}\right]
$$

The Second Level IHS transform add color details to the fused images. Color information is an important factor while we considering the visual quality of the image. Also to improve the spectral quality the original Hue and Saturation components of the MS image is fused with the intensity component of the previously fused bands $\left\{\mathrm{R}_{\mathrm{IHS}}^{\prime \prime}, \mathrm{G}_{\mathrm{IHS}}^{\prime \prime}, \mathrm{B}_{\mathrm{IHS}}^{\prime \prime}\right\}$. The fused bands transformed HIS[11] domain,

$$
\left[\begin{array}{c}
\mathrm{I}^{\prime \prime} \\
\mathrm{H}^{\prime \prime} \\
\mathrm{S}^{\prime \prime}
\end{array}\right]=\operatorname{HSI}\left(\mathrm{R}_{\mathrm{IHS}}^{\prime \prime}, \mathrm{G}_{\mathrm{IHS}}^{\prime \prime}, \mathrm{B}_{\mathrm{IHS}}^{\prime \prime}\right)
$$

Use Hue and Saturation of the original MS images and the intensity component of the fused image are used to find the final fused MS images.

$$
\left[\begin{array}{l}
R_{f} \\
G_{f} \\
B_{f}
\end{array}\right]=\text { inverse_HSI }\left(I^{\prime \prime}, H, S\right)
$$

\section{Experimental Analysis}

To evaluate our method we selected QuickBird and IKONOS satellite images which have $4 \mathrm{~m}$ resolution for MS bands and $1 \mathrm{~m}$ resolution for PAN image. For testing the quantization level reduced from 11 bits to 8 bits. Since there are no reference images are available, we use the original MS images as reference images and scale down the fused MS images and PAN image to the size of reference image for the quantitative analysis.

In order to evaluate the performance and effectiveness of our proposed fusion method, we compared the new algorithm with five other existing fusion methods; IHS, modified IHS by Choi, NSCT+ IHS, Improved-IHS and Adaptive IHS. From the images in Fig. 1 and Fig. 2 we can observe that the IHS produces the color distortions. The modified IHS, IKONOS IHS and improved IHS by Choi are fused the images with less spectral distortions but the spatial and visual qualities are very poor. The NSCT and IHS combined method produce low spatial and visual quality images but the spectral quality is good, also the computation time for the fusion process is very high as compared with all other methods. Proposed method produce good spatial and visual quality images with less spectral distortions than IHS. To provide quantitative comparison of different fusion algorithms, we use eight objective indicators shown in Table 1.

Here SSIM[12] is used as a quantitative measure for the visual quality. The PAN image is considered as the perfect quality image. The RGB image is converted to grayscale and compared with the PAN image. When more structural details are preserved in the fused image; better the visual quality. Spectral distortion is calculated using three different measures; ERGAS[13], RASE[14] and SAM, while spatial quality is measured using Cross Correlation (CC) and Universal Image Quality Index (UIQI)[15]. Cross 
correlation and UIQI are calculated for each band with PAN image. In order to analyze the performance of our method is achieve a good spectral and spatial quality levels along with visual quality, the sum of difference between the spatial quality improvement and spectral distortions, and SSIM is calculated, shown in Table 1, Eq. No 8. Since RASE is calculated as rate of distortion; CC and SSIM is calculated as the rate of spatial and visual quality improvement, higher values of the QDIFF will represent the good performance of the algorithm. Table 2, shows the quantitative analysis of the IKONOS and QuickBird images.

Table 1. Quantitative metrics

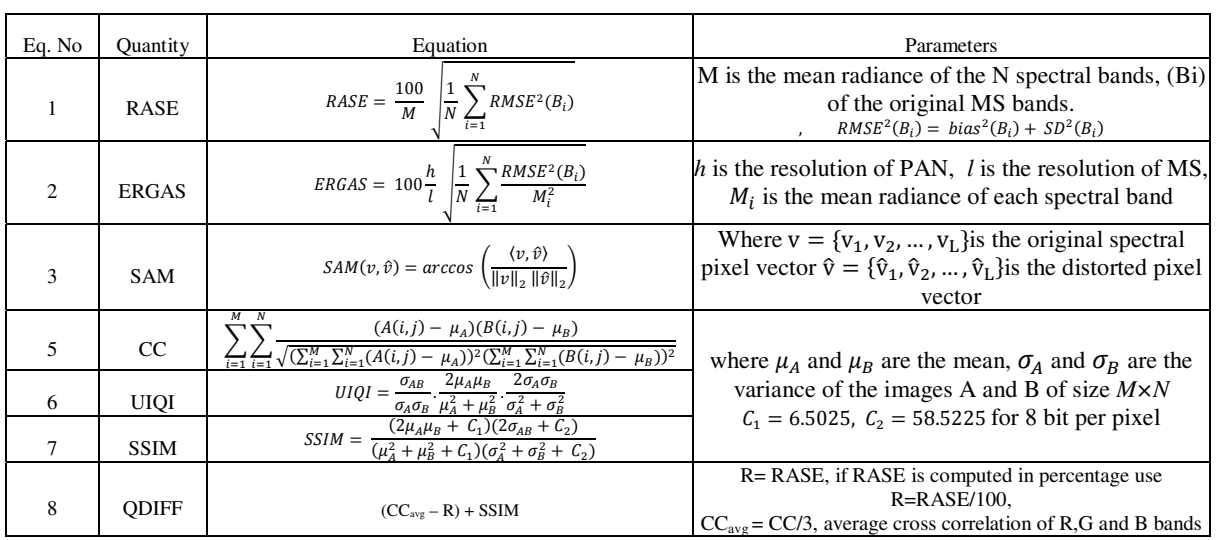

Subjective analysis is carried out to compare the visual quality of the fused image, using $1000 \times 1000$ IKONOS images which are fused using the same six techniques used in quantitative analysis. Subjects are asked to rank the six images from 1 to 6 (1 is considered as best) based on visual quality (sharpness, color, brightness, contrast, overall quality); people are selected from variety of domains such as students, image processing experts, industry etc. The result of subjective analysis is shown in Table 3. From the table we can understand that most of the people ranked the proposed method as best and IHS as the bad image.

Table 2. Quantitative analysis of sample IKONOS and QuickBird images $(1000 \times 1000)$

\begin{tabular}{|l|c|c|c|c|c|c|c|c|c|c|c|c|}
\hline & \multicolumn{1}{|l|}{ IKONOS 1000x1000 image } & \multicolumn{3}{|l|}{ QuickBird 1000x1000 image } \\
\hline & IHS & Proposed & Chol & NSCT + IHS & Ada IHS & Imp IHS & IHS & Proposed & Chol & NSCT + IHS & Ada IHS & Imp IHS \\
\hline RASE & 45.2460 & 38.7459 & 51.4174 & 4.8126 & 65.0229 & 23.8175 & 26.9824 & 18.3354 & 10.2818 & 4.2225 & 7.8227 & 8.3906 \\
\hline ERGAS & 11.5157 & 8.4085 & 9.3753 & 1.2025 & 15.5509 & 6.0714 & 6.7561 & 4.3637 & 2.5127 & 1.0570 & 1.9686 & 2.0988 \\
\hline SAM & 3.0302 & 0.9945 & 3.0253 & 0.8287 & 2.6654 & 1.7284 & 1.5648 & 0.7269 & 0.9317 & 0.7029 & 0.7947 & 0.8977 \\
\hline UIQI Red & 0.5120 & 0.6417 & 0.9491 & 0.5405 & 0.5508 & 0.7316 & 0.9588 & 0.9733 & 0.9554 & 0.9218 & 0.9171 & 0.9066 \\
\hline UIQI Green & 0.6154 & 0.7378 & 0.9551 & 0.5963 & 0.6326 & 0.7511 & 0.9721 & 0.9750 & 0.9394 & 0.9148 & 0.9104 & 0.9019 \\
\hline UIQI Blue & 0.4070 & 0.5233 & 0.8520 & 0.4166 & 0.4484 & 0.5507 & 0.9712 & 0.9693 & 0.9435 & 0.9122 & 0.9073 & 0.9012 \\
\hline CC Red & 0.9808 & 0.8849 & 0.8883 & 0.3831 & 0.8933 & 0.4244 & 0.9938 & 0.9708 & 0.9412 & 0.8777 & 0.8848 & 0.8659 \\
\hline CC Green & 0.9921 & 0.9394 & 0.9076 & 0.4244 & 0.8914 & 0.4834 & 0.9968 & 0.9822 & 0.9433 & 0.8752 & 0.8773 & 0.8624 \\
\hline CC Blue & 0.9722 & 0.8000 & 0.8301 & 0.1566 & 0.8120 & 0.1887 & 0.9933 & 0.9683 & 0.9246 & 0.8465 & 0.8506 & 0.8352 \\
\hline SSIM & 0.6856 & 0.7632 & 0.8199 & 0.4500 & 0.7058 & 0.5174 & 0.9716 & 0.9540 & 0.8795 & 0.7446 & 0.7802 & 0.7556 \\
\hline QDIFF & 1.2148 & 1.2505 & 1.1811 & 0.7232 & 0.9212 & 0.6447 & 1.6964 & 1.7444 & 1.7131 & 1.5689 & 1.5728 & 1.5262 \\
\hline Time (sec) & 0.2899 & 1.1126 & 0.1870 & 244.0583 & 1.5189 & 0.2647 & 0.1608 & 1.1074 & 0.2230 & 232.6192 & 1.1569 & 0.2031 \\
\hline
\end{tabular}




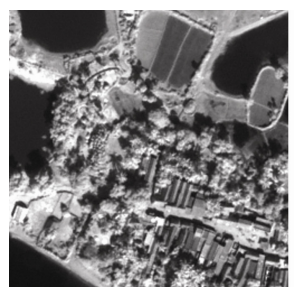

(a)

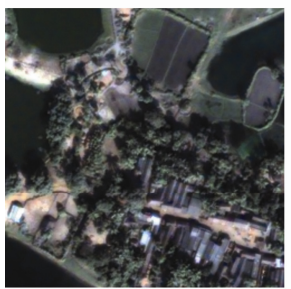

(e)

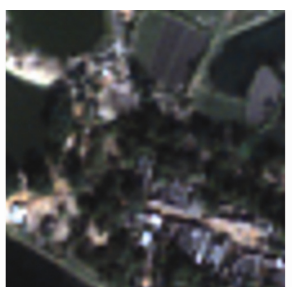

(b)

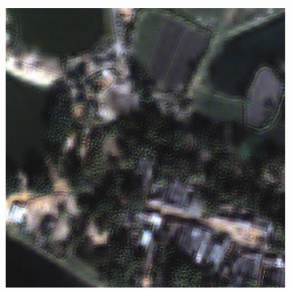

(f)

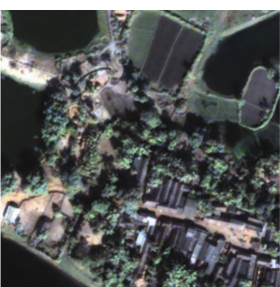

(c)

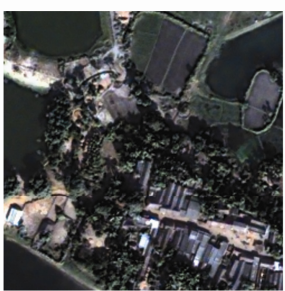

(g)

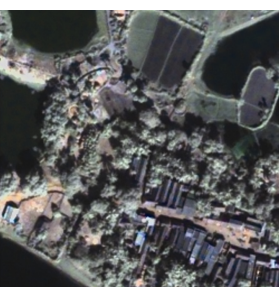

(d)

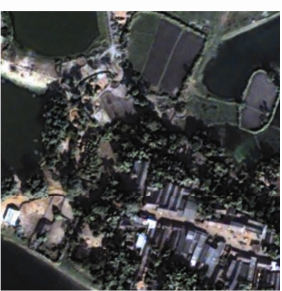

(h)

Fig. 1. QuickBird fused images (a) Panchromatic (b) Scaled up Multispectral RGB(c) Proposed (d) IHS (e) modified IHS by Choi (f) NSCT+ IHS (g) Improved-IHS (h) Adaptive IHS

Table 3. Subjective analysis results of sample IKONOS image $(1000 \times 1000)$

\begin{tabular}{|l|c|c|c|c|c|c|}
\hline & IHS & Proposed & Choi & NSCT + IHS & Ada IHS & Imp IHS \\
\hline Avg Ranks & 5.00 & 2.00 & 4.60 & 2.90 & 3.20 & 3.30 \\
\hline
\end{tabular}

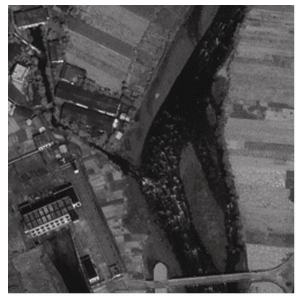

(a)

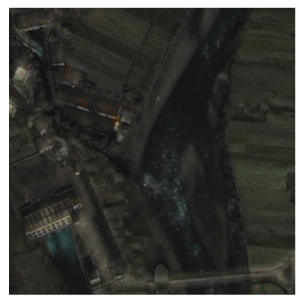

(e)

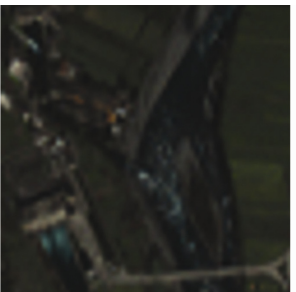

(b)

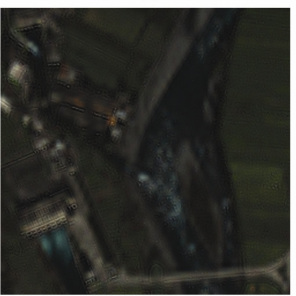

(f)

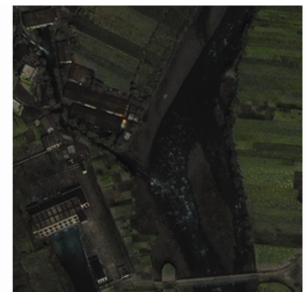

(c)

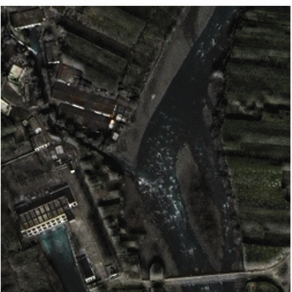

(g)

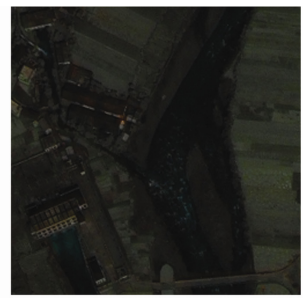

(d)

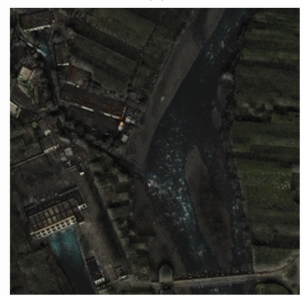

(h)

Fig. 2. IKONOS fused images (a) Panchromatic (b) Scaled up Multispectral RGB(c) Proposed (d) IHS (e) modified IHS by Choi (f) NSCT+ IHS (g) Improved-IHS (h) Adaptive IHS

\section{Conclusion}

In this work, a Bi-Level IHS image fusion algorithm for satellite images is proposed. From the quantitative and qualitative analysis, proposed method demonstrates good visual and 
spatial qualities with reduced spectral distortion. Our method balances the spatial and spectral quality while improving the visual quality of the fused satellite images. The method is computationally fast and it can be implemented on a parallel computing architecture.

\section{References}

1. Haydan, R., Dalke, G.W., Henkel, J., Bare, J.E.: Applications of the IHS color transform to the processing of multisensor data and image enhancement. In: Proceedings of the International Symposium of Remote Sensing of Arid and Semi-arid Lands, Cairo, Egypt, pp. 599-616 (1982)

2. Eshtehari, A., Ebadi, H.: Image Fusion of Landsat ETM+ and Spot Satellite Images Using IHS, Brovey and PCA. Toosi Univ. Technol., Tehran (2008)

3. da Cunha, A.L., Zhou, J., Do, M.N.: The nonsubsampled contourlet transform: Theory, design, and applications. IEEE Trans. Image Process. 15(10), 3089-3101 (2006)

4. Shah, V.P., Younan, N.H., King, R.L.: An Efficient Pan-Sharpening Method via a Combined Adaptive PCA Approach and Contourlets. IEEE Transactions on Geoscience and Remote Sensing 46(5), 1323-1335 (2008)

5. Mahyari, A.G., Yazdi, M.: Panchromatic and Multispectral Image Fusion Based on Maximization of Both Spectral and Spatial Similarities. IEEE Transactions on Geoscience and Remote Sensing 49(6) (June 2011)

6. Li, D.: Fusion of Multispectral Remote Sensing Image and High Resolution Spatial Panchromatic image Based on NSCT and IHS. In: Second International Conference on Computer and Electrical Engineering (2009)

7. Kaplan, N.H., et al.: Fusion of multispectral and panchromatic images by combining bilateral filter and HIS transform. In: 20th European Signal Processing Conference (FUSIPCO 2012), Bucharest, Romania, August 27-31, pp. 2501-2505 (2012)

8. Rahmani, S., Strait, M., Merkurjev, D., Moeller, M., Wittman, T.: An Adaptive IHS PanSharpening Method. IEEE Transactions on Geoscience and Remote Sensing 7(4), 746-750 (2010)

9. Choi, M., Kim, H., Cho, N.I., Kim, H.O.: An improved intensity-hue-saturation method for IKONOS image fusion. Korea Adv. Inst. Sci.Technol., Daejon, Korea. Tech. Rep. 06-9 (2008)

10. Choi, M.: A New Intensity-Hue-Saturation Fusion Approach to Image Fusion With a Tradeoff Parameter. IEEE Transactions on Geoscience and Remote Sensing 44(6), 1672-1682 (2006)

11. Gonzalez, R.C., Woods, R.E.: Digital Image Processing, 3rd edn. Pearson Prentice Hall (2009)

12. Wang, Z., Bovik, A.C., Sheikh, H.R., Simoncelli, E.P.: Image quality assessment: From error visibility to structural similarity. IEEE Transactions on Image Processing 13(4), 600-612 (2004)

13. Wald, L.: Data Fusion: Definitions and Architectures Fusion images of different spatial resolutions. Les Presses, EColedes Mines De Paris (2002)

14. Alperone, L., et al.: Comparison of pansharpening algorithms, Outcome of the 2006 GRS S data Fusion contest. IEEE Trans. Geoscience and Remote Sensing 45(10), 3012-3021 (2007)

15. Wang, Z., Bovik, A.C.: A universal image quality index. IEEE Signal Process. Lett. 9(3), 81-84 (2002) 